\title{
Design of Simulation Competition Platform Based on Cognitive Behavior Modeling
}

\author{
Junjie Zeng, Qi Zhang*, Yunxiu Zeng, Long Qin, Mei Yang, Quanjun Yin \\ College of Systems Engineering, National University of Defense Technology, Changsha 410073, Hunan Province, China \\ *Corresponding author: Qi Zhang, zhangqiy123@126.com
}

\begin{abstract}
Cognitive behavior modeling of agent is an important component of simulation system, and there are some difficulties in the simulation of course teaching. When students make simulation experiments about cognitive behavior modeling, such as algorithm design and model construction, there is no simulation competition platform that is controllable, flexible and scalable. To solve this problem, we propose a simulation competition platform based on cognitive behavior modeling, called TankSim, for undergraduate and graduate students. This platform aims to cultivate student's team collaboration and innovation capability, and improve their learning motivation. This paper elaborates the proposed platform from three aspects, including demand analysis, platform design, and content design.
\end{abstract}

Keywords: Simulation discipline; Design of the competition platform; Cognitive behavior modeling; Agent

Publication date: August 2021; Online publication: August 30, 2021

\section{Introduction}

The scientific experiment is an important way to understand and change the world, but in many cases, it is not suitable or impossible to conduct experiments directly on real systems because it is risky, expensive, and difficult to control experimental conditions as well as the fact that the real system has not been established yet. In order to solve the above-mentioned problems, simulation was proposed and became the third way to understand and change the world after theoretical research and experimental research. The process of simulation ${ }^{[1]}$ that is based on similar principles establish a model that reflects the characteristics of the real system, which can be implemented as an operational system to carry out experimental research, personnel training, program demonstration and other activities.

In the simulation system, there are autonomous or semi-autonomous virtual agents that are generated and controlled by computers. They are used to model equipment, facilities, personnel and other entities in order to reflect various complex behaviors of entities in environments ${ }^{[2]}$. The core task of constructing agent is to model its behaviors, whose essence is to model human behavior. Human behavior modeling can be divided into two categories, namely physical behavior modeling and cognitive behavior modeling ${ }^{[3]}$. Physical behavior modeling uses physical principles to model and verify the direct interaction process between entities and environments. Cognitive behavior modeling models the command capabilities of entities based on the internal thinking or mental processes of the brain.

In order to cultivate innovative and high-quality talents, various universities and regions across the country vigorously carry out various competitions. In the field of simulation, the most representative national-level important competition is the "National Computer Simulation Grand Prix." The competition is co-sponsored by Higher Education Department of the Ministry of Education, Chinese Association of Automation, China Computer Users Association, China Simulation Federation, and Chinese Institute of Command and Control. It is organized by the "Computer Integrated Manufacturing Systems" magazine ${ }^{[4]}$. 
It plays an important role in popularizing computer simulation knowledge and promoting the cultivation of innovation talents in the field of simulation. The competition focuses on evaluating the participants' abilities to model the whole simulation process, including simulation platform construction, model development, and visualization. Due to the characteristics of the Simulation Grand Prix, it is unable to provide a unified platform for cognitive behavior modeling and construct a community-style competition environment.

In order to solve the above-mentioned problems, we propose TankSim, a simulation competition platform based on cognitive behavior modeling. The platform is independently developed and easy for customization. The participants are mainly for college students and graduate students. The platform adheres to the design concept of "innovative collaboration, open sharing, education in competition, and research promotion via competition." It integrates cutting-edge technologies, such as modeling and simulation, artificial intelligence, cloud computing, and intelligent human-computer interaction, providing a public open platform for competitive confrontation, exchange and sharing, and academic research. Through this competition platform, students can carry out research on key issues, such as agent planning and decisionmaking, situational awareness, knowledge reasoning, confrontation games, and collaborative cooperation. Besides, this simulation platform can effectively cultivate their team collaboration and innovation capabilities and improve their simulation modeling level, and can be pragmatic in the scientific and technological research and engineering practice foundation. In addition, the competition platform can be used as an extended experimental environment for simulation courses. It can improve students' interests toward course learning by typical case traction and group confrontation, and stimulate students' enthusiasm for learning.

\section{Demand analysis}

For college students, through in-depth understanding and use of the platform, they will become familiar with cognitive behavior modeling in the simulation system, understand the cutting-edge artificial intelligence algorithms, and be proficient in applying the professional knowledge of simulation and related disciplines, thereby improving their own collaborative innovation ability and stimulating the enthusiasm of learning. For graduate students, after using the platform, they will master the relevant methods of cognitive behavior modeling, apply cutting-edge artificial intelligence algorithms proficiently, and propose excellent algorithms for cognitive behavior modeling, thereby improving their engineering practice ability and scientific research skills.

The competition platform proposed in this paper focuses on the field of agent's cognitive behavior modeling. According to technologies in cognitive science, knowledge engineering, artificial intelligence and other fields, modeling methods can be roughly divided into four categories: modeling methods based on knowledge engineering, modeling methods based on cognitive structure, modeling method based on planning decision theory, and modeling methods based on machine learning. The modeling methods based on knowledge engineering are used in most simulation systems for cognitive behavior modeling. This kind of method collects domain expert knowledge for specific tasks and then constructs behavior models that fully meets the expectations of experts. The modeling methods based on cognitive structure mainly reflect the application results of researchers in exploring the cognitive structure of the brain. The modeling methods based on the planning decision theory adopt the decision-making method of artificial intelligence to approximate the cognitive decision-making process, and its core is to determine the goals and criteria of intelligent decision-making, and to solve them as a rational and optimal problem. The modeling methods based on machine learning use the learning method to autonomously generate and optimize the behavior model ${ }^{[5]}$. The proposed platform currently supports the behavior tree method that is a modeling method based on knowledge engineering commonly used in the industry, and the modeling methods based on 
machine learning that is of great concern to the academic community.

In order to facilitate students to construct the cognitive behavior model, this platform mainly supports the following functions: behavior tree editing, import and operation based on JSON format, behavior model construction based on machine learning, custom creation of agent and its initial state, importing and exporting the agent initial state, setting of simulation times, playback of simulation results, setting of simulation speedup, simulation control, display of simulation situation, map custom editing, map switching, and etc.

\section{Competition platform design}

The competition platform proposed in this paper is based on $\mathrm{C}++$ and consists of four projects, namely Basic project, GridMapEditor project, MyBevTree project, and VisTankSim project.

The Basic project is mainly responsible for implementing the basic classes that support the operation of the platform. It mainly includes: (i) classes and functions about the dynamic generation and execution of behavior trees; (ii) classes and functions about simulation parameter settings, result playback, and etc.; (iii) classes and functions about agent's state initialization, state update, perception of environmental data and execution of actions; (iv) classes and functions about network communication. This project is used to generate a static library for the VisTankSim project to call.

The GridMapEditor project is a map editor for supporting platforms developed based on the singledocument MFC (Microsoft Foundation Classes) standard. Students can generate a customized map by the editor. Specifically, the editor supports the following functions: (i) create a blank map; (ii) generate or remove obstacles by clicking with the mouse; (iii) save the current map; (iv) import an existing map.

The MyBevTree project is used to implement external precondition classes of a custom behavior tree. After students use the JSON editing tool to complete the writing of the behavior tree, they need to develop custom external preconditions based on $\mathrm{C}++$ in the project. This project is used to generate a static library for the VisTankSim project.

The VisTankSim project is used to develop executable programs based on the single-document MFC standard that supports platform visualization operations. This project uses the static library generated by the Basic project and the MyBevTree project to realize the display and control of the simulation situation.

Since methods based on knowledge engineering are adopted by most simulation systems at present, this platform provides an interface for constructing the cognitive behavior model based on the method of knowledge engineering. Common methods based on knowledge engineering mainly include rule systems, finite state machines, decision trees, Petri nets, and behavior trees. The rule system is an important method of cognitive behavior modeling in the traditional system. It has the advantages of simple development and strong expressive ability. However, there is no clear organizational structure between the rules, and its model complexity increases rapidly with the number of rules. Finite state machines have the advantages of easy implementation and strong interpretability, but as the number of states increases, there will be shortcomings, such as difficulty in maintenance and poor scalability ${ }^{[6]}$. Decision tree has the advantages of modularity and strong hierarchy, but its expression is redundant and difficult to maintain. Petri net has the advantage of being good at describing and publishing behavior, but it is difficult to be mastered by nonprofessionals as it is highly abstract ${ }^{[7]}$. The behavior tree not only retains the advantages of easy development and strong interpretability, but also has the advantages of modularization and strong scalability. In recent years, behavior trees have become a mainstream method of cognitive behavior modeling in the fields of games, robots and simulation ${ }^{[8,9]}$.

By comparing and analyzing the advantages and disadvantages of the above-mentioned methods, this platform decides to provide students with a scalable and easy-to-develop behavior tree method. Specifically, the platform has developed control nodes, action nodes, and external precondition nodes of the behavior 
tree. The control nodes include priority selection nodes, random selection nodes, parallel nodes, and sequence nodes. Students can directly call the control nodes and action nodes provided by the platform. Since the external precondition node involves the specific behavioral logic of agent, the platform only implements the base class of the external precondition node, and students are required to inherit the base class to develop a custom external precondition node.

With the rapid development of artificial intelligence technology, the authenticity of the cognitive behavior model has been improved, and more and more researchers try to apply machine learning methods to the field of cognitive behavior modeling. Considering that most machine learning algorithms are implemented based on languages, including python, this platform has improved the network communication interface to communicate with other languages. Specifically, this platform uses the Boost library to implement socket communication, which can send the perceived data and attributes of the agent and the message data sent between the agent in byte stream format, and receive the action data in byte stream format. In addition, the network communication interface implemented by this platform facilitates the deployment of machine learning algorithms on servers with powerful computing capabilities in the same local area network.

\section{Competition content design}

The proposed competition platform is based on the idea of "action traction, platform support." It takes the offensive and defensive battles of tank as the main background, centers on typical tasks such as advancing, unfolding, and seizing impact targets, designs tanks encounter battle as competition scenarios, and promotes competition activities. In the competition scenarios, the red and blue tanks meet at a certain place and are demanded to end the battle within the specified time. In this scenario, both sides carry a certain number of missiles to attack each other, and can open shields to defend against missiles. Tanks are equipped with a series of sensors to detect different elements on the map separately, and the tanks can communicate internally.

Tanks mainly perceive the environment through sensors. There are six types of sensors: obstacle sensors, risky sensors, radar sensors, radar wave sensors, sniffing sensors, and ultrasonic sensors. Among them, the obstacle sensor is used to sense whether the grids of the front, rear, left, and right of the tank are blocked; the risky sensor is used to sense incoming artillery shells from the front, back, left, and right at any distance; the radar sensor is used to perceive the information of all objects within 3 grid widths in front of the tank; the radar wave sensor is used to sense whether there are radar signals from other tanks in the front, rear, left and right directions; the sniffing sensor is used to sense the distance of the nearest tank; the ultrasonic sensor is used to perceive the movement of the nearest tank at previous step. The directions of tank's movements include forward, backward, left, right, and it can turn on and off the radar, set the power of the radar, turn on and off the shield, fire, and send messages to other tanks. However, moving (forward or backward) and turning (left or right) cannot be performed at the same time.

Students participating in the competition can write scripted behavior models based on behavior trees, or they can write adaptive behavior models based on machine learning algorithms. Using behavior trees to develop cognitive behavior models can be divided into two steps:

(1) Adopt JSON editing tools to compile behavior trees in a specified format, and use control nodes to organize the overall logic of the model. Leaf nodes must be action nodes. Both control nodes and action nodes can be mounted with external precondition nodes, which are used to determine whether to execute the control node or action node.

(2) Implement the external precondition nodes of the custom behavior tree by programming. The input of external preconditions, including perception data and its own attributes, long-term memory for 
communication between nodes and messages sent by other agents, is on short-term memory.

In order to facilitate students to use machine learning to build the cognitive behavior model, this platform provides network communication functions and agent classes written in python:

(1) main() is the main function, including connection to the competition platform, tank initialization, receiving state data, and generating decision-making behavior;

(2) connectServer(), which uses socket to connect to the server;

(3) receiveGameInf(), which receives platform-related information published by the server;

(4) receiveAllData(), which receives all agent perception data, attribute data and communication data within agents;

(5) sendAllOutputs(outputs), which sends all agent action information to the platform;

(6) class agent, through which students can implement machine learning related functions in the class.

\section{Conclusions}

With the rapid development of information technology, simulation has become an important method for mankind to understand and change the world. It plays an increasingly important role in the scientific research, equipment testing, and training of various personnel in various industries. Modeling the cognitive behavior is an important part of the construction of simulation systems, and it is also a significant and difficult point in cultivating professional simulation technical personnel. Since course teaching merely provides students with a comprehensive understanding of cognitive behavior modeling issues, it is important to guide students to explore and practice independently based on their existing knowledge by a simulation platform. The design of the platform is to increase students' enthusiasm for learning in simulation disciplines, tap students' potential, and improve students' collaborative innovation capabilities through competitions, which has impressive effects on cultivating simulation talents. In future, the competition platform will be optimized in the following three aspects: (i) upgrade from local deployment to cloud deployment, so that students can upload their own design behavior models to the cloud server to fight against others at any time; (ii) an improvement of the model architecture to support more modeling methods; (iii) an increase of the number of default scenarios and creation of an interface for scenario editing.

\section{Acknowledgments}

We appreciate the fruitful discussion with the Sim812 group.

\section{Funding}

Natural Science Foundation of Hunan Province (Project number: 2017JJ3371).

\section{Disclosure statement}

The authors declare that there is no conflict of interest.

\section{Author contributions}

Conceptualization, Q.Z. and Y.Z.; Data curation, L.Q.; Formal analysis, Q.Z. and M.Y.; Funding acquisition, Q.Y. and M.Y.; Investigation, J.Z., and Q.Z.; Project administration, Q.Y.; Validation, J.Z. and Y.Z.; Writing original draft, J.Z.; Paper review \& editing, J.Z. and L.Q. 


\section{References}

[1] Huang K, 2010, Modeling and Simulation Technology. National University of Defense Technology Press, 1-2.

[2] Guo Q, 2006, Introduction to Computer Generated Forces. National Defense Industry Press.

[3] Huang K, Liu B, Huang J, 2004, Review of Combat Simulation Technology. Journal of System Simulation, 16(9): 1887-95.

[4] “Computer Integrated Manufacturing Systems" Editorial Department. National Computer Simulation Grand Prix, http://www.jsjfz.com/award.html

[5] Galway L, Charles D, Black M, 2008, Machine Learning in Digital Games: A Survey. Artificial Intelligence Review, 29(2): 123-61.

[6] Yang R, Huang X, 2011, Computer-generated Force Intelligent Decision-Making Method and its Application. Publishing House of Electronics Industry.

[7] Murata T, 2010, Petri Nets: Properties, Analysis and Applications. Proc IEEE, 77(4): 541-80.

[8] Rabin S, 2014, AI Game Programming Wisdom 4. Nelson Education.

[9] Sekhavat YA, 2017, Behavior Trees for Computer Games. International Journal on Artificial Intelligence Tools, 1: 1-27. 